\title{
EchoGéo
}

36 | 2016

Stratégies de villes et "modèles urbains"

\section{Circulation des politiques urbaines et internationalisation des villes : la stratégie des relations internationales de Johannesburg}

\section{Elisabeth Peyroux}

\section{(2) OpenEdition}

Journals

Electronic version

URL: https://journals.openedition.org/echogeo/14623

DOI: $10.4000 /$ echogeo. 14623

ISSN: 1963-1197

Publisher

Pôle de recherche pour l'organisation et la diffusion de l'information géographique (CNRS UMR 8586)

Electronic reference

Elisabeth Peyroux, "Circulation des politiques urbaines et internationalisation des villes : la stratégie des relations internationales de Johannesburg", EchoGéo [Online], 36 | 2016, Online since 30 June 2016, connection on 10 August 2021. URL: http://journals.openedition.org/echogeo/14623 ; DOl: https://doi.org/10.4000/echogeo.14623

This text was automatically generated on 10 August 2021.

EchoGéo est mis à disposition selon les termes de la licence Creative Commons Attribution - Pas d'Utilisation Commerciale - Pas de Modification 4.0 International (CC BY-NC-ND) 


\title{
Circulation des politiques urbaines et internationalisation des villes : la stratégie des relations internationales de Johannesburg
}

\author{
Elisabeth Peyroux
}

L'internationalisation des villes fait l'objet d'une littérature abondante qui transcende les frontières disciplinaires. Les travaux de géographie économique, géographie urbaine, études urbaines, science politique, sociologie politique, et plus récemment les relations internationales, se sont intéressés aux différentes formes que prend ce processus dans la période contemporaine en mobilisant différentes conceptualisations de la ville. Les écrits sur l'internationalisation des économies urbaines ont souligné la place centrale des villes, notamment dites "globales", dans la mondialisation économique en tant que lieux d'accumulation des richesses et moteurs de croissance dans le contexte de développement du capitalisme (Sassen, 2001 ; Knox et Taylor, 1995). Les recherches récentes sur l'internationalisation des politiques urbaines ont donné une place centrale aux villes dans les processus de «mobilité » des politiques au-delà des frontières nationales en tant que nœuds d'échanges et de savoirs dans le cadre de l'accélération de la transnationalisation des politiques et des normes (Peck et Theodore, 2010; McCann et Ward, 2011). Enfin, les travaux sur l'internationalisation des villes et des gouvernements locaux (Pinson et Vion, 2000 ; Viltard, 2008) conçoivent les villes comme des acteurs internationaux qui gagnent de l'influence sur la scène internationale dans les domaines de la diplomatie, de l'aide au développement et de la coopération, notamment à travers l'émergence de formes de gouvernance en réseaux transnationaux venant bousculer les fondations d'un système international basé sur la prééminence des États et des organisations internationales (Nijman 2009; Acuto, 2013; Bouteligier, 2014 ; Curtis, 2014 ; Lingkvist, 2014). Cette internationalisation des villes est activement construite à travers des stratégies de développement économique et de marketing urbain pour renforcer la compétitivité et la visibilité internationale des villes, de policy tourism pour identifier et importer de «bonnes pratiques 
internationales » (McCann, 2013), de « diplomatie urbaine » pour répondre à des enjeux locaux ou globaux et renforcer l'influence des villes dans la gouvernance mondiale (van der Pluijm 2007, Viltard 2008).

Cet article vise à enrichir les débats sur les stratégies d'internationalisation des villes d'une part en croisant les apports des travaux sur la «mobilité des politiques urbaines" et les questionnements relatifs à l'« action internationale des villes", d'autre part en intégrant une clé de lecture géopolitique des relations entre villes.

3 En effet, bien qu'ayant pour objet une réflexion commune sur l'action des villes à l'échelle internationale, ces champs dialoguent peu entre eux. Les chercheurs qui travaillent sur la «mobilité des politiques urbaines » et sur les coopérations entre villes ne se sont pas saisis de deux enjeux théoriques importants pour analyser la production, diffusion et circulation de normes et politiques urbaines. Le premier a trait à la reconfiguration des relations entre villes et États, qui est au cœur des débats théoriques en science politique, politique comparée, sociologie politique et relations internationale portant sur «l'action internationale» des villes et des collectivités locales (Pinson et Vion, 2000; Viltard, 2008, 2010; van der Pluijm, 2007) et leur "capacité d'action» à l'échelle internationale (Acuto 2013; Bouteligier, 2014; Lungkvist, 2014), car elle met en question la pertinence des cadres de pensée fondés sur des catégories étatiques et interétatiques. Le second enjeu renvoie au rôle des réseaux transnationaux et à la manière dont cette forme de gouvernance confère aux villes des ressources, des moyens et des positions d'influence et de pouvoir, notamment pour produire et diffuser des normes internationales (Bouteligier, 2014).

4 Par ailleurs, en privilégiant les enjeux de compétitivité économique dans l'analyse des stratégies d'internationalisation des villes, l'ensemble de ces travaux prend insuffisamment en compte le rôle des enjeux géopolitiques et la manière dont les circulations actuelles participent de la recomposition des hiérarchies urbaines. Les relations entre villes se construisent dans un cadre géopolitique changeant, dont il est important de saisir les enjeux et les implications. L'emploi du terme " géopolitique » ne renvoie pas à des conflits à proprement parler, ni à des rivalités sur des territoires nationaux ou locaux en lien avec des questions de souveraineté mais à des rivalités en termes de positionnement international dans un espace de référence géopolitique (Nepad - New Partnership for Africa's Development-, Brics - Brésil, Russie, Inde, Chine, Afrique du Sud - pour les villes du Sud). Ces relations de compétition peuvent se jouer au sein d'espaces qui peuvent être immatériels (alliances et réseaux internationaux de villes, forums) (Acuto, 2013). Cette dimension géopolitique est à replacer dans le cadre de la montée en puissance économique mais également diplomatique des pays de la zone des Brics (Véron, 2013) et des débats autour des nouvelles normes et paradigmes de développement qu'induiraient ces rapports Sud/ Sud (Mawdsley, 2012a et b). La production de la ville s'élabore en effet dans le cadre d'une relation d'échanges et d'emprunts, et ce de plus en plus dans le cadre Sud/Sud (Verdeil, 2005 ; Bénit-Gbaffou et al., 2012 ; Didier et al., 2012 ; Morange et al., 2012).

5 Cet article analyse la stratégie des relations internationales de Johannesburg mise en œuvre par le gouvernement métropolitain (City of Johannesburg Metropolitan Municipality) depuis 2012. Celle-ci vise à renforcer les coopérations entre villes et la participation de Johannesburg à des réseaux transnationaux en développant les échanges et transferts d'expériences et de «bonnes pratiques». Elle vise aussi à consolider la position économique et à promouvoir une plus grande visibilité de 
Johannesburg à l'échelle régionale, continentale et internationale. L'analyse s'appuie sur des entretiens conduits à Johannesburg en 2011 et 2013', sur une analyse de contenu du document politique adopté en 2012 (City of Johannesburg - CoJ -, 2012) et sur la collecte et l'analyse d'informations et de documents à propos des échanges et des partenariats entre villes publiés sur le site Internet de la ville de Johannesburg².

6 En adoptant la perspective d'une ville du Sud, cet article répond à l'appel visant à « décentrer » notre regard de chercheur et à prendre en considération les innovations politiques, les liens et les flux circulant au sein du Sud (Robinson, 2011). Il rend compte des nouvelles positions de ces villes sur la scène internationale et de la manière dont cela s'accompagne de nouvelles catégorisations et hiérarchisation.

7 La première partie de l'article présente l'état des lieux de la recherche sur l'internationalisation des villes et la circulation des politiques urbaines en soulignant les apports, les limites et les enjeux analytiques et théoriques. La seconde partie analyse la stratégie de Johannesburg en questionnant la nature des relations entre la ville et l'État. L'analyse montre l'étroite imbrication des objectifs économiques et géopolitiques en lien avec l'agenda géopolitique de l'État sud-africain. Ces objectifs économiques et politiques conditionnent le cadre dans lequel s'effectuent la production, diffusion et circulation de normes, expertises et politiques urbaines en orientant la sélection des villes partenaires et en déterminant le contenu et les modalités des échanges. L'article montre que la stratégie internationale de Johannesburg contribue à l'intensification de la circulation des politiques urbaines issues des expériences des villes du Sud et participe à la consolidation des relations au sein du continent africain et entre les villes des Brics. Si ces relations peuvent représenter un moyen de promouvoir des formes alternatives de développement urbain, la catégorisation dont elles font l'objet contribue à produire des formes collaboratives mais néanmoins hiérarchiques au sein desquelles le critère de performance économique joue un rôle sélectif et déterminant.

\section{Villes, État et relations internationales}

8 Les travaux sur l'internationalisation des villes ont contribué au renouvellement de la conceptualisation des villes en tant que lieux et acteurs et à la manière dont elles s'insèrent dans un système de relations à diverses échelles. Issus de différentes perspectives disciplinaires et cadres d'analyse, ils ont pour point commun une remise en question du rôle de l'État et un questionnement sur l'importance à accorder aux territoires dans les processus étudiés.

\section{Internationalisation des économies et des politiques urbaines}

9 Les écrits sur l'internationalisation des économies urbaines se sont intéressés aux relations économiques entre les villes et les firmes économiques, saisies à travers les flux de capitaux, la mise en place d'infrastructures et de technologies de communication (Derruder et al., 2007), au sein d'un «système-monde » mettant en relation des réseaux de villes (Knox et Taylor, 1995). Ils ont mis en lumière les limites d'une pensée d'une part centrée sur l'État, d'autre part sur le territoire (Derruder et al., 2007). Ces travaux souffrent toutefois d'un biais géographique (en se focalisant sur les villes du Nord), hiérarchique (en accordant un statut supérieur aux villes du Nord) et 
économique (en négligeant les autres dimensions à l'œuvre dans les processus de mondialisation) (Pirie, 2010 ; Curtis, 2014).

Les publications récentes sur l'internationalisation ${ }^{3}$ des politiques urbaines dans le champ de la géographie et des études urbaines critiques se sont emparées de ces autres dimensions en s'intéressant à la mobilité des idées, normes, pratiques et politiques urbaines. S'inscrivant dans une approche ouverte et relationnelle de la ville (Massey, 2005), elles ont montré comment les villes sont constituées à travers leurs relations avec d'autres lieux et d'autres échelles et comment ces relations entre villes façonnent leurs trajectoires de développement (Jabobs, 2012 ; Söderström et al., 2013 ; Söderström, 2014 ; McCann et Ward, 2010 ; Robinson, 2011). Elles ont appelé à des études urbaines plus cosmopolites et à un comparatisme Sud/Sud permettant de dépasser les cadres d'analyse hérités de la pensée occidentale (Parnell et Robinson, 2012). Ces travaux ont renouvelé les analyses classiques de science politique sur les «transferts de politiques publiques » en remettant en cause plusieurs présupposés : la linéarité du transfert, la rationalité des choix et des pratiques, et le rôle prépondérant des États dans les processus de transfert (Peck et Theodore, 2010). Dénonçant le «territorialisme méthodologique " d'une grande partie des études urbaines (McCann et Ward, 2012, p.44), ils proposent de prendre en considération dans l'étude de la production des villes les géographies relationnelles et territoriales, les mouvements, les flux et les ancrages, le rôle des contextes globaux et des enjeux locaux et territoriaux (McCann et Ward, 2010).

\section{L'action internationale des villes et des collectivités locales}

11 Les travaux sur l'action internationale des villes en science politique et relations internationales se sont saisis d'un enjeu peu traité par les travaux cités précédemment. Il invite à questionner la capacité d'action des villes sur la scène internationale à travers les nouvelles relations de pouvoir et d'influence qui se nouent entre l'État et des acteurs infranationaux et transnationaux non étatiques de plus en plus nombreux dans le champ des relations internationales, de la diplomatie et de la gouvernance mondiale (Acuto, 2013). Outre des activités de partenariats interurbains développés de longue date, les villes mettent en œuvre des stratégies de communication, d'échanges, de positionnement et de lobbying au sein de réseaux, alliances de villes et forums transnationaux (C40, United Cities and Local Governments - UCLG -, Cities for Climate Protection program - CCP -, International Council for Local Environmental Initiatives ICLEI), qui sont devenus aujourd'hui non seulement des lieux d'échanges d'expertise et de «bonnes pratiques » mais aussi des lieux stratégiques de production et de diffusion de normes internationales, à l'image de la gouvernance environnementale (Betsill et Bulkeley, 2006). Les villes entretiennent également des interactions directes avec des organisations régionales et internationales (Unicef, Unesco, UN-Habitat) et collaborent sur la définition de lois internationales et de politiques globales (Nijman, 2009).

12 L'hypothèse d'un accroissement de l'influence des villes sur la scène internationale s'appuie sur le constat d'une "urbanisation des défis globaux» (Ljunkvist, 2014, p. 154) : les villes et les questions urbaines acquièrent une place de plus en plus centrale et pertinente dans la régulation des grands problèmes contemporains (ibid., p. 52; Acuto, 2013). Dans un contexte où la mondialisation contribuerait à éroder les responsabilités et fonctions de l'État (van der Pluijm, 2007), les villes, reconnues comme 
des acteurs de la mondialisation tout en étant affectées par elle (ibid., p. 8), sont considérées comme les mieux placées pour répondre aux enjeux globaux, notamment dans le cadre de la lutte contre les effets du changement climatique (Bouteligier, 2014).

Ces pratiques remettent en cause certains présupposés des études en science politique et relations internationales sur le rôle majeur des États dans les relations internationales (diplomatie, politiques étrangère) et le fait que les gouvernements infranationaux soient placés sous la seule influence et direction des gouvernements nationaux (Acuto, 2013; Bouteligier, 2014). Faisant écho aux études urbaines, les chercheurs invitent à dépasser le " piège territorial des relations internationales » et à considérer les autres modalités spatiales de la politique mondiale, tels que les réseaux, les flux et le place making (Acuto, 2013, p. 23 ; Agnew, 2015).

Ces enjeux théoriques sont explorés à partir de plusieurs perspectives. Un premier ensemble de publications en science politique et relations internationales s'est intéressé plus particulièrement à la notion de "diplomatie urbaine" (Van Pluijm, 2007 ; Viltard 2008, 2010) et questionne "l'action internationale » ou "extérieure » des collectivités locales, envisagée principalement sous l'angle de leur rapport à l'État. La diplomatie, définie comme la représentation de la ville et de ses intérêts (van der Pluijm, 2007 ; Viltard, 2008), est conçue comme une «forme de décentralisation de la gestion des relations internationales" (van der Pluijm, 2007, p.11). Les questions portent sur les causes, motivations, formes et contenus des engagements (bilatéraux ou multilatéraux), sur la nature des relations entre les gouvernements centraux et les collectivités locales (compétition, coopération, complémentarité, débordement, remplacement) (Viltard, 2008 et 2010 ; van der Pluijm, 2007). Elles s'accompagnent d'hypothèses sur la fragmentation du pouvoir étatique, la perte de souveraineté des États vis-à-vis des gouvernements locaux et des nouveaux acteurs transnationaux et la "décomposition du système westphalien " (Viltard, 2008, p. 511). Les réseaux sont ici définis comme des « scènes diplomatiques " (Viltard, 2010, p. 599).

Le second sous-ensemble est consacré à une réflexion sur le rôle des villes dans les affaires internationales et la gouvernance mondiale (global governance) et traite plus directement de la capacité d'action politique de la ville (political agency of city) en tant que lieux et acteurs (Acuto, 2013, p. 5). Il fait le lien entre les analyses en termes de société en réseaux et celles portant sur les villes globales en questionnant le rôle et la capacité d'action des villes, des maires et des réseaux transnationaux (Bouteligier, 2014). Il s'interroge plus particulièrement sur les sources et les impacts de cette agentivité (ibid.), sur la manière dont les réseaux permettent aux villes d'acquérir de l'influence et du pouvoir sur la scène internationale (Curtis, 2014 ; Bouteligier, 2014) et dans quelle mesure ces derniers participent à la création de nouvelles «sphères d'autorité » (Betsill et Bulkeley, 2006, p. 154).

Les chercheurs qui travaillent sur la "mobilité des politiques » et sur les coopérations entre villes ne se sont pas saisis de ces enjeux théoriques ${ }^{4}$. L'attention des premiers s'est portée sur les acteurs de la mobilité, du transfert, de l'adoption et de la réappropriation de ces politiques "en mouvement ", souvent resitués dans le contexte de néolibéralisation des politiques urbaines (Ward, 2006 ; Peyroux et al., 2012 ; Didier et al., 2012; Wood, 2014 ; Michel et Stein, 2015). D'autres publications ont privilégié une analyse relationnelle des villes et le rôle de la circulation internationale des politiques urbaines dans leur trajectoire de développement (Söderström, 2014). Les seconds se sont intéressés à la construction historique des partenariats transmunicipaux (Clarke, 
2012), aux relations entre collectivités territoriales et États dans la coopération décentralisée (Pasquier, 2012), à l'efficacité des partenariats interurbains (Bontenbal, 2009) et à leur rôle comme vecteurs d'intégration européenne (Pinson et Vion, 2000) ou comme lieu d'apprentissage (Campbell, 2009 ; van Ewijk et Baud, 2009).

\section{Enjeux analytiques et conceptuels}

17 Ces travaux manquent de clarté conceptuelle: la notion "d'acteurs " englobe indifféremment les villes dans leur ensemble ou les collectivités locales sans tenir compte du caractère complexe et hétérogène de la ville, de la diversité de ses intérêts et de ses représentations (Acuto, 2013). En réalité, beaucoup de publications traitent des stratégies d'internationalisation des collectivités locales, de leurs représentants, fonctionnaires ou élus, notamment du maire (Beal et Pinson, 2014 ; Barber 2013) ou des réseaux ou alliances qui les représentent (UCLG par exemple ${ }^{5}$ ). Cependant, ils ne rendent pas compte des divergences de positionnement et d'intérêt des villes au sein des réseaux et alliances et de la manière dont cela affecte leur capacité d'influence. La question des réseaux mérite d'être traitée de manière différenciée afin de tenir compte de ceux composés d'une grande variété d'acteurs publics et privés (à l'image de Cities Alliance, qui inclut des représentants de gouvernement locaux, des gouvernements nationaux, des organisations non gouvernementales et des organisations multilatérales comme la Banque mondiale et UN-Habitat). De la même façon, le terme de diplomatie " urbaine » apparaît trop englobant : la distinction entre jumelages, coopérations entre villes et activités au sein de réseaux transnationaux est importante à faire, car ces activités renvoient à différentes généalogies, contextes et temporalités. Elles s'inscrivent dans des dynamiques institutionnelles et politiques différentes et renvoient, comme nous l'avons vu, à des débats différents sur le rôle politique des villes.

Ces travaux restent pour l'heure peu fondés sur des résultats empiriques démontrant réellement le pouvoir et/ou l'influence de ces villes, deux dimensions qui ne sont pas toujours clairement distinguées. Certains écrits font référence à une "présence urbaine" au sein de la gouvernance mondiale (Acuto, 2013, p.138) ou à une " présence active des villes et de leurs gouvernements locaux dans le champ de la politique mondiale, des affaires internationales et de la gouvernance mondiale " (Lungqvist, 2014, p.11) en soulignant qu'il reste encore à fournir des preuves empiriques qu'elles "agissent" à l'échelle internationale (ibid.). Par ailleurs, les conclusions générales, qui tendent à montrer que les États et les organisations internationales conservent leur pouvoir et prérogatives (Viltard, 2008), qui indiquent un déplacement de la prédominance des États-nations plutôt qu'un remplacement des gouvernements centraux par les villes (Acuto 2013), ou à l'inverse un contournement des États par les villes (Nijman, 2009) demandent à être confrontées à des études de terrain tenant compte de la diversité des cadres institutionnels inter-gouvernementaux (Kübler et Piliutyte, 2007), mais aussi, comme nous le verrons, de la convergence ou non des stratégies des villes et des États.

19 Enfin, l'ensemble de ces travaux ne prend pas suffisamment en compte le rôle des enjeux géopolitiques dans les stratégies d'internationalisation des villes et la circulation internationale des politiques urbaines. Les publications sur le rôle international des villes s'en tiennent souvent à des discours généraux sans ancrage 
empirique - voir toutefois Acuto (2013) pour son analyse du réseau C40. Si la dimension géopolitique est traitée dans le cadre des partenariats interurbains (mouvements municipaux transnationaux, coopérations décentralisées, jumelages) (Clarke, 2012), elle l'est le plus souvent dans une perspective historique et européenne. La montée en puissance économique, politique et diplomatique des "pays émergents ", des nouvelles alliances et constellations Sud/Sud, notamment entre les Brics (Véron, 2013 ; Harrison 2015), n'est pas prise en compte, alors que les travaux dans le champ de la géographie et du développement montrent que les "nouveaux donneurs" issus des économies émergentes prônent une approche différente voire alternative à celles des bailleurs de fonds dits " traditionnels ${ }^{6}$ dans le champ de l'aide au développement et la coopération (Braude et al., 2008; Kragelund, 2011; Mawsdley, 2012a et b). Cette «nouvelle géographie du développement » invite à s'interroger sur la manière dont les stratégies de production et diffusion de normes et politiques par les villes à l'échelle internationale s'articulent à celles des États et contribuent ou non à la production de normes alternatives communes.

\section{La stratégie des relations internationales de Johannesburg : l'affirmation d'une stratégie autonome alignée sur les objectifs, priorités et valeurs du gouvernement central}

20 La capacité d'action de Johannesburg sur la scène internationale implique de s'interroger en premier lieu sur les relations entre la ville et l'État : ici, l'influence de la ville sur la scène internationale ne doit pas se comprendre en termes de rivalité mais d'alignement et de renforcement mutuel des objectifs économiques, politiques et géopolitiques des échelons de pouvoir nationaux et locaux.

\section{Gouvernements locaux et relations internationales dans le contexte de Johannesburg}

21 La sortie de l'isolement diplomatique de l'Afrique du Sud en 1994, la levée des sanctions internationales et des boycotts dont elle avait fait l'objet depuis les années $1960^{7}$, et les nouvelles législations sur les gouvernements locaux ont permis à ces derniers de développer et formaliser leurs activités de coopération internationale. Dans le contexte sud-africain de décentralisation du système de gouvernement, les relations internationales relèvent des trois "sphères" (ou «tiers") nationale, provinciale et locale. Établies par la constitution de 1996 comme « distinctes », « interdépendantes » et "inter-reliées", elles sont régies par un impératif de coopération intergouvernementale. Les gouvernements locaux peuvent développer des relations internationales sous deux conditions : celles-ci doivent les aider à atteindre les objectifs que leur fixe la constitution; elles doivent être mises en œuvre en collaboration avec les deux autres sphères du gouvernement. Elles répondent donc à un objectif interne contribuer au développement économique local et au renforcement des capacités de gestion des villes en lien avec leur stratégie de développement et de croissance - et à des objectifs externes - renforcer les liens de l'Afrique du Sud avec les autres pays et sa position internationale conformément aux grandes orientations nationales ${ }^{8}$. 
22 À Johannesburg, les activités internationales se sont tout d'abord effectuées dans le cadre d'une "politique municipale des relations internationales" adoptée par le gouvernement central en 1999 (The Municipal International Relations Policy Framework - MIR) (Villiers, 2005; Ruffin 2013ํ). L'importance du jumelage avait été reconnue par le gouvernement national sud-africain et l'African National Congress (ANC) au pouvoir qui y voyait un moyen de contribuer au développement des capacités locales des gouvernement et de promouvoir une image positive de la nouvelle Afrique du Sud (Villiers, 2005). La MIR, qui représentait un cadre incitatif et non contraignant pour les municipalités, est définie comme « un lien entre deux communautés ou plus issues de deux États-Nations différents, au sein duquel l'un des acteurs-clés est une municipalité. Ces liens peuvent inclure des organisations non gouvernementales, des organisations à base communautaire ou des associations privées »(DPLG, 1999, p. 3, cité in Villiers, 2005, p. 248). Une confusion sur la nature des acteurs-clés domine toutefois (ibid.). L'avant-propos du texte politique met en effet l'accent sur le rôle des municipalités: la MIR "promeut des partenariats entre des municipalités sudafricaines et des municipalités à travers le monde de façon à assurer un apprentissage maximum, une synergie et une promotion de nos intérêts nationaux, y compris la promotion de l'investissement » (ibid., p. 248). L'exemple de Johannesburg montre qu'il s'agit avant tout de relations entre municipalités. À la fin des années 2000, la Municipalité de Johannesburg était ainsi engagée dans plusieurs jumelages avec des gouvernements locaux du Nord et du Sud et s'était engagée dans deux partenariats visant un transfert d'expertise en vue de renforcer les capacités locales des Municipalités d'Addis-Abeba (Éthiopie) et de Lilongwe (Malawi) ${ }^{10}$.

En 2012, face aux critiques de la MIR, formulées au sein de la Municipalité de Johannesburg en raison de sa mise en œuvre limitée, de sa dimension uniquement cérémonielle et du manque de coordination entre les acteurs ${ }^{11}$, la Central Strategy Unit (CSU) a développé sa propre "stratégie des relations internationales» (SRI) (COJ, 2012) ${ }^{12}$. Cette unité, qui est placée sous l'autorité du Bureau du maire exécutif, est chargée de la planification stratégique, de la gestion des performances et de la mise en œuvre de la stratégie de développement et de croissance à long terme de Johannesburg (Growth and Development Strategy - GDS - 2040). Le pouvoir métropolitain, institué en 2001 suite aux réformes territoriales et administratives des structures héritées de l'apartheid, affirmait la volonté de consolider la stratégie, de la rendre plus efficace que la précédente ${ }^{13}$. Fondée sur une réévaluation des partenariats existants, elle institue une méthode de sélection des villes partenaires en fonction de critères économiques et géopolitiques et propose un programme d'actions clair, structuré autour d'objectifs bien définis, avec des résultats attendus concrets et mesurables. Quatre types d'activités sont identifiés: les «engagements de villes à villes»(jumelages, collaboration autour de projets, mentoring et "programmes entre pairs»), le « réseautage » (networking), les « relations inter-gouvernementales» et la " gestion du savoir et l'apprentissage ", montrant ici une maîtrise de l'usage des technologies de l'information et de la communication.

\section{La préservation d'une autonomie municipale}

24 Cette stratégie montre un alignement sur les objectifs, priorités et valeurs du gouvernement central et provincial plutôt qu'une collaboration formelle entre les différentes sphères de gouvernement. La Municipalité opère en réalité dans un «vide 
institutionnel $\aleph^{14}$, qui lui permet de dégager une marge d'autonomie. Le Département des Relations Internationales et de la Coopération (DIRCO) 2010-2013 et le Plan national de développement (NDP, Vision for 2030), qui fixent les orientations politiques et stratégiques du gouvernement national, ne fournissent en effet pas de lignes directrices sur le rôle que doivent jouer les municipalités dans le positionnement international de l'Afrique du Sud (CoJ, 2012, p. 57) : l'intégration verticale des activités internationales des trois sphères n'y est pas traitée. Il revient donc à la Municipalité de Johannesburg, par la nature et la teneur de ses engagements internationaux, de refléter les objectifs, priorités et valeurs des gouvernements national et provincial, la présence du parti de l'ANC à la tête du gouvernement national et de la Municipalité de Johannesburg facilitant la convergence des approches. La composante «relations intergouvernementales » développée dans le cadre de la SRI de Johannesburg vise à combler ces manques institutionnels en assurant un meilleur alignement des activités de chacun des acteurs institutionnels. La Municipalité de Johannesburg affiche ainsi le souci "d'intégrer le rôle des villes [...] dans l'agenda national en tant qu'une composante essentielle pour atteindre les objectifs de croissance et de développement de la région » (CoJ, 2012, p. 62).

Pour l'heure, la mise en œuvre concrète de la stratégie reste du ressort de la Municipalité sous la houlette de la Central Strategy Unit (CSU). Elle implique un petit nombre de fonctionnaires des différents départements ou d'élus, y compris le maire, qui initient et participent aux visites sur le terrain (study tours), à la signature d'accords de coopération, à la réalisation ou à l'accompagnement de projets dans les villes partenaires en privilégiant les coopérations entre municipalités - et non directement entre communautés. Elle vise toutefois une ouverture aux acteurs privés dans un souci de tirer des bénéfices économiques des échanges entre villes, notamment avec les Brics ${ }^{15}$ ). Les partenariats des années 1990 et 2000 en Afrique du Sud représentaient un instrument d'amitié et d'échanges inscrit dans le contexte de la solidarité internationale à l'égard de la lutte contre l'apartheid et de la transition démocratique. Ils sont devenus un outil de renforcement des capacités gestionnaires ${ }^{16}$. Les nouvelles orientations soulignent l'intérêt marqué pour les intérêts commerciaux dans le cadre des échanges entre villes.

La stratégie s'appuie sur un puissant outil de marketing urbain, le Jike (Jo'burg Innovation \& Knowledge Exchange), qui s'occupe de l'accueil des délégations étrangères en visite à Johannesburg. Il promeut et diffuse son expertise à l'échelle nationale et internationale sous la forme de newsletters (Knowledge Insight), de brochures et autres synthèses qui offrent une vitrine des « bonnes pratiques » de Johannesburg en matière de gestion et de planification urbaine identifiées par la Municipalité, ancrées dans sa transition estimée « réussie » vers un pouvoir métropolitain unifié (1997-2001) : " The Johannesburg Transformation Story " $)^{17}$.Cette transition a pourtant fait l'objet de vives contestations à l'époque en raison de son caractère dirigiste et contraignant, et de son orientation entrepreneuriale et néolibérale (Didier et al., 2012). Cette stratégie est renforcée par les activités du Visitor and Resource Centre (V\&RC), ouvert en 2002 par le maire de Johannesburg.

La stratégie dispose de moyens financiers limités : elle n'a pas de budget propre; les financements des déplacements sont pris en charge les départements concernés par les thématiques des échanges. Mais elle s'appuie sur le soutien financier, technique et la médiation de réseaux nationaux (The South African Local Government Association - 
Salga) et internationaux de collectivités locales (UCLG), d'organismes internationaux (Banque mondiale) et d'agences de coopération européennes (GIZ), notamment dans le cadre de ses partenariats avec Addis-Abeba ${ }^{18}$ ).

\section{Catégorisation et hiérarchisation des villes dans les pratiques de coopérations bilatérales et d'insertion dans les réseaux transnationaux}

La SRI de Johannesburg est conçue comme un prolongement de la stratégie de développement de la Municipalité (GDS 2040). Le partage d'expériences entre villes doit permettre d'améliorer la gouvernance, l'administration et la fourniture de services de Johannesburg et celles des villes partenaires en vue de répondre aux objectifs de développement fixés par la GDS 2040 (Peyroux, 2015) et, plus largement, de contribuer à la croissance et au développement du continent africain. La stratégie s'appuie sur une catégorisation distincte des villes africaines et des villes des Brics.

Dans un contexte de compétition reconnue avec les villes émergentes de l'Afrique de l'Ouest, la stratégie vise à répondre à l'ambition de la GDS 2040 de positionner Johannesburg comme une "World Class African City of the future » (CoJ, 2012, p. 6). En tant que capitale régionale de la SADC et « hub économique stratégique », elle entend affirmer un profil de "ville leader » et préserver son « avantage compétitif » à l'échelle du Gauteng, de la SADC, et du continent africain (ibid., p. 10). En qualité de nouvel entrant parmi les villes des Brics (2010), Johannesburg vise également à occuper une position stratégique au sein des économies émergentes en développent un "branding " actif de la ville et du pays tout entier « en tant que ville leader du pays » (ibid., p. 8).

Les villes partenaires prioritaires sont identifiées et sélectionnées sur la base de leur intérêt stratégique pour Johannesburg et l'Afrique du Sud. Cet intérêt se fonde sur une analyse détaillée des tendances internationales en matière d'urbanisation, de développement économique et de croissance, et des enjeux qu'elles représentent pour les villes dans un environnement mondialisé. Les critères de sélection des villes partenaires sont géographiques (localisation), économiques (poids, taille, potentiel de croissance démographique, atouts sectoriels) et géopolitiques (priorité accordée aux relations Sud/Sud).

\section{La consolidation d'une position de leadership au sein du continent africain}

31 Les échanges avec les villes africaines s'inscrivent dans un objectif de soutien à la croissance et au développement des villes, dans le cadre de l'intégration régionale et du Nouveau partenariat pour le développement de l'Afrique (Nepad). Elle se fonde sur une compréhension relationnelle des économies urbaines et régionales, considérées comme interdépendantes dans le contexte de mondialisation économique, particulièrement au sein du continent africain (l'exemple de l'immigration de populations africaines en Afrique du Sud fuyant des contextes politiques instables ou la pauvreté est cité). Les villes sélectionnées dans le cadre de la stratégie actuelle occupent une position stratégique au sein de l'économie régionale et continentale : ce sont des villes jouant un rôle dans les « corridors » et autres « clusters » économiques, structurant les processus 
de développement régionaux, ou des villes au fort potentiel de développement démographique et économique (Luanda, Lagos, Maputo).

Partager des expériences de politiques économiques et urbaines permet, selon Johannesburg, de «réduire la vulnérabilité des espaces urbains vis-à-vis du changement global » (ibid., p. 6). Le renforcement des compétences des gouvernements locaux, notamment en Afrique, de leur capacité de «fonctionnement " est vu comme un impératif pour "récolter les bénéfices de la croissance économique et de l'urbanisation » (ibid.). Johannesburg se met en position d'apporter une assistance à des villes africaines considérées comme moins bien placées qu'elle pour répondre aux conséquences de l'urbanisation rapide, de l'étalement urbain, de la croissance des quartiers informels et de la persistance de la pauvreté et de l'inégalité, en raison de la faiblesse de leurs capacités gestionnaires. Cette attention portée à l'Afrique s'inscrit dans les priorités du gouvernement central qui entend «répondre aux besoins développementaux des pays en développement ». Les accords de coopération passés ont ainsi pris la forme d'un programme de mentoring avec les villes d'Addis-Abeba et de Lilongwe dans le cadre de la préparation de leur stratégie de développement. Bien plus qu'un simple transfert de compétences, la participation de Johannesburg, par l'ampleur de son travail au sein de la Municipalité de Lilongwe (entretien 1, 20 septembre 2011), montre en réalité une forme de co-construction de politiques locales.

Ces partenariats entre villes représentent un moyen de diffuser les normes et les valeurs portées par Johannesburg et l'Afrique du Sud. Ainsi la ville de Johannesburg promeut-elle à travers ses échanges avec les villes africaines une politique "pro-poor ", qui doit, selon elle, s'attaquer à la persistance des inégalités que la montée des classes moyennes ne saurait occulter (ibid., p.7). Elle défend le principe de participation publique dans l'élaboration des politiques (comme ce fut le cas dans le partenariat avec Lilongwe) (entretien 1, 20 septembre 2011). Elle affiche une volonté d'indépendance financière par rapport aux bailleurs de fonds internationaux afin de garder un esprit critique (ibid.). Elle conteste l'action des «agences de développement qui guident les agendas de développement des villes africaines et échouent à prendre en compte la complexité des besoins de [nos] villes » (COJ, 2012, p. 7).

\section{Un rapprochement avec les villes des Brics}

Les villes des Brics sont reconnues de leur côté comme des "villes globales du futur ", des "hubs de compétitivité » appelés à jouer un rôle de plus en plus important et influent sur la scène internationale (COJ, 2012, p. 8). Celles-ci sont associées à des capacités de résilience face à la crise de 2008 , à des transformations importantes de leur structure économique (notamment la montée des classes moyennes) et à des efforts en matière de développement technologique et d'infrastructures (ibid.). Johannesburg reconnaît qu'elle est dotée de moins d'atouts que les autres (en termes de taille de population, d'indice de développement humain). Aussi, elle vise à développer des relations avec les villes considérées comme les plus dynamiques et les plus innovantes (Sao Paulo, New Delhi, Shanghai) dans le cadre de «relations entre pairs " (peer to peer) qui apportent des bénéfices mutuels, notamment dans les secteurs des technologies de l'information et de la communication (Chine), du logement (Brésil) et du textile (Inde) ${ }^{19}$. La stratégie de Johannesburg vient consolider l'agenda politique du gouvernement à l'égard des Brics, jugé encore peu développé par la ville, en 
contribuant à positionner l'Afrique du Sud en tant que «catalyseur » de relations Sud/ Sud, un objectif de l'ANC (CoJ, 2012, p. 54). Johannesburg s'inscrit dans la ligne politique de l'ANC qui, dans son Discussion Document on International Relations (2012), considère que le soutien apporté à l'alliance avec les Brics représente « une plateforme pour des idées alternatives au consensus de Washington et aux politiques néolibérales" (ibid., p. 54). La ville promeut le rôle des gouvernements locaux dans la fourniture " universelle » de services publics en soulignant que les stratégies financières ne peuvent se centrer uniquement sur des partenariats public/privé et doivent se tourner davantage vers des municipalités élues (ibid., p. 45). La nature de cette "alternative » au néolibéralisme reste toutefois vivement débattue (Didier et al., 2012 ; Parnell et Robinson, 2012).

Dans un contexte de diminution des moyens financiers alloués aux jumelages par des villes du Nord affaiblies par la crise de 2008, et de changement des besoins de Johannesburg, les relations Nord/Sud ne sont plus prioritaires (ibid., p. 9). Depuis l'achèvement de son processus de restructuration en métropole unifiée (1997-2001), Johannesburg ne se considère plus dans une phase d'apprentissage dans les domaines de coopération traditionnels tels qu'observés dans le cadre des partenariats avec des villes comme Birmingham, Londres et New York (gestion financière, régénération urbaine et sécurité) (entretien 3, 29 septembre 2011). Elle estime que les partenariats doivent désormais être "smart»: des bénéfices tangibles sont attendus dans le domaine de la santé, de la transition vers une stratégie bas-carbone dans le champ de l'aménagement urbain, de l'économie verte et des «smart cities» (CoJ, 2012, p. 67). Un partenariat privilégié avec Bilbao et New York est programmé en raison de leur statut de capitales régionales et de leurs pratiques innovantes en matière de "smart cities « (ibid., p. 80).

Le tissage de relations privilégiées avec les villes des Brics souligne un changement de perception des hiérarchies urbaines de la part de la Ville de Joburg: si la stratégie internationale requérait, il y a une dizaine d'années, de se positionner comme "ville globale durable » par rapport aux «big cities» ou "alpha cities» du "premier monde » comme Londres ou New York, l'émergence économique des villes des Brics a changé la donne (entretien 3, 29 septembre 2011).

\section{Une participation active aux réseaux et alliances transnationaux}

Les activités de "réseautage» («networking») occupent une place importante dans cette stratégie. Elles sont même considérées comme les activités les plus importantes (entretien 2, 21 octobre 2013) en raison de la vitrine internationale et des occasions qu'elle offre à Johannesburg. La participation à des évènements-phares comme les conférences et les forums ( $\mathrm{C} 40$, Metropolis) offre à Johannesburg un cadre dans lequel présenter, et donc médiatiser et diffuser ses «bonnes pratiques» en matière de planification, de gestion urbaine et de gouvernance. Ces pratiques ont porté leurs fruits : sa participation à la Cop 21 en décembre 2015 à Paris a été couronnée par un prix du C40 Cities Award pour son leadership dans la lutte contre le changement climatique (Green Bond initiative). C'est suite à une présentation de sa City Development Strategy que Johannesburg a été directement sollicitée par Cities Alliances, UCLG et Salga pour jouer le rôle de "mentor " auprès de la ville de Lilongwe afin de l'aider à développer sa propre stratégie de développement. Ce partenariat a d'ailleurs été récompensé par un prix en 2011 décerné par Metropolis, UCLG et un gouvernement 
municipal chinois ${ }^{20}$ (entretien 1, 20 septembre 2011). Les réseaux de gouvernements locaux créent en effet des occasions de partenariat entre villes : UCLG facilite la mise en relation des villes et la réplication des «bonnes pratiques» (CoJ, 2012, p. 62). Ainsi le programme de mentoring a-t-il été étendu à d'autres villes sud-africaines, au Malawi, au Mozambique et à la Namibie (entretien 1, 20 septembre 2011). Johannesburg se construit une position de leadership en matière de gestion métropolitaine : Bamako lui a récemment demandé de l'assister dans le domaine de la gestion des déchets, de l'électrification rurale et de l'eau ${ }^{21}$.

La capacité d'action de Johannesburg est associée à la capacité d'influencer la production de normes et les pratiques urbaines. Johannesburg privilégie à ce titre la participation à des évènements susceptibles de "forger les opinions publiques » et d'avoir un impact sur les perceptions («opinions formers») : évènements «à fort contenu » («content-events ») plutôt que des «méga-évènements » ${ }^{22}$ (entretien 2, 21 octobre 2013). Ainsi la ville a-t-elle accueilli la réunion annuelle de Metropolis en 2013 Caring cities, qui a rassemblé 500 participants, et en 2014 la $5^{\mathrm{e}}$ biennale de C40, rassemblant des maires du monde entier. Elle a activement participé à la Cop 17 à Durban en 2011, où le maire de Johannesburg, alors président de Salga, a utilisé cette tribune pour faire valoir la position des citoyens du Gauteng et nourrir la politique nationale ${ }^{23}$.

La ville donne ainsi la priorité aux réseaux considérés de " haut niveau » (Metropolis, UCLG), à des réseaux ou organismes qui promeuvent l'intégration régionale et les relations Sud/Sud (l'Union africaine, Africities, UCLGA, Brics Friendship Cities and Local Government Forum) et aux forums qui promeuvent les smart cities, l'innovation et l'économie verte (C40, ICLEI). Elle fait du lobbying pour l'élection du maire, des membres du comité du maire ou du City Manager à des positions-clés. Johannesburg, membre du ICLEI, a ainsi accueilli son secrétariat et l'un de ses maires a été membre du bureau international. Son maire actuel est membre du comité de pilotage du C40 et coprésident de Metropolis. Johannesburg entend par ailleurs jouer un rôle de lobbying fort auprès des organisations basées au Nord pour faciliter l'intégration de la ville dans des réseaux soutenant les pratiques relatives aux smart cities.

\section{Conclusion}

Les travaux récents en géographie, études urbaines, sociologie politique et science politique ont enrichi les réflexions sur les transferts, la mobilité et la circulation internationale des politiques urbaines en lien avec un renouveau des approches conceptuelles de la ville. L'analyse de la construction du rôle politique des villes à l'échelle internationale, ici saisies dans leur dimension de pouvoir institutionnalisé, peut s'enrichir de son côté des débats théoriques issus du champ des relations internationales. La ville, ou plutôt l'urbain, s'il se généralise dans ses formes et ses processus de déploiement spatial, capturant et instrumentalisant de plus en plus d'espaces, y compris lointains et marginaux (Brenner 2013; Brenner et Schmid, 2015) étend en effet également son emprise dans le champ politique. Cela se traduit par la manière dont les grands enjeux sont formulés aujourd'hui (les défis globaux deviennent des questions urbaines) (Ljungkvist 2014), par la manière dont l'échelle des solutions est pensée (les villes comme acteurs locaux et internationaux) et dont les formes organisationnelles d'une partie croissante de la gouvernance mondiale sont promues 
(réseaux ou alliances de villes transnationaux). En étendant leur champ de compétences dans la production, diffusion et circulation de normes et de politiques à l'échelle internationale, les villes modifient les équilibres et les rapports de force hérités du système westphalien, un enjeu dont la géographie et les études urbaines doivent se saisir pour comprendre les nouvelles dynamiques politiques transnationales. La théorisation des villes comme acteurs internationaux, en tant « qu'agents collectifs » ou «agents en réseaux» (Acuto, 2013) reste toutefois à renforcer par des études empiriques qui font encore défaut et un travail conceptuel et théorique interdisciplinaire à approfondir.

41 En montrant comment les stratégies urbaines sont fortement liées aux enjeux géopolitiques, notamment dans le cadre de la montée en puissance des économies émergentes, l'exemple de Johannesburg éclaire la manière dont les catégories et hiérarchies de villes se recomposent aujourd'hui à partir du Sud. Cette étude encore incomplète invite à rapprocher les travaux sur la «nouvelle géographie du développement " (Mawsdey, 2012) de ceux traitant de la "nouvelle géographie de la gouvernance mondiale » (Acuto, 2013) afin d'approfondir l'étude de leurs positions sur la scène internationale dans une perspective qui se doit non seulement de dépasser les clivages traditionnels Nord/Sud mais aussi d'explorer les divergences au sein des villes du Sud.

\section{BIBLIOGRAPHY}

Acuto M., 2013. Global Cities, Governance and Diplomacy. The Urban Link. Routledge, London, 220 p.

Agnew J., 2015. Revisiting The Territorial Trap, Nordia Geographical Publications, 44, 4, p. 43-48.

Barber B.J., 2013. If Mayors Ruled the World: Dysfunctional Nations, Rising Cities. Yale University Press, $432 \mathrm{p}$.

Beal V., Epstein R., Pinson G., 2015. La circulation croisée. Modèles, labels et bonnes pratiques dans les rapports centre-périphérie. Gouvernement et action publique, 3, p. 103-127.

Beal V., Pinson G., 2014. When Mayors Go Global: International Strategies, Urban Governance and Leadership. The International Journal of Urban and Regional Research, 28, 1, p. 302-317.

Bénit-Gbaffou C., Didier S., Peyroux E., 2012. Circulation of security models in Southern African cities: Between neoliberal encroachment and local power dynamics, numéro spécial The International Journal of Urban and Regional Research, 36 (5), p. 877-889.

Betsill M., Harriet B., 2006. Cities and the Multilevel Governance of Global Climate Change. Global Governance, 12, 2, p. 141-159.

Bontenbal M., 2009. Strengthening urban governance in the South through city-to-city cooperation: Towards an analytical framework. Habitat International, 33, 2, p. 181-189.

Bouteligier S., 2014; A Networked Urban World. Empowering Cities to Tackle Environmental Challenges. In Curtis S. (ed), The Power of Cities in International Relations. London and NewYork, Routledge, p. 57-68. 
Braude W., Pearl T., Sidiropoulos E., 2008. Emerging donors in international development assistance: the South Africa case. Research Report, International Development Research Centre, Ottawa.

Brenner, N. (ed), 2013. Implosions/Explosions. Towards a study of planetary urbanization. Berlin, Jovis, $576 \mathrm{p}$.

Brenner N., Schmid C., 2015. Towards a new epistemology of the urban? City, 19, 2-3, p. 151-182.

Campbell T., 2009. Learning cities: Knowledge, capacity and competitiveness. Habitat International, 33, p. 195-201.

City of Johannesburg, 2012. An integrated International Relations Agenda for the City of Johannesburg. Group Strategy, Policy Coordination and Relations. City of Johannesburg.

Clarke N., 2012. Urban policy mobility, anti-politics, and histories of the transnational municipal movement. Progress in Human Geography, 31, (1), p. 25-43.

Curtis S. (ed), 2014. The Power of Cities in International Relations. London and NewYork, Routledge, $192 \mathrm{p}$.

Derudder B., Witlox F., Taylor P.J., 2007. Les villes dans les réseaux mondiaux : une nouvelle méthodologie pour cartographier la position relationnelle des villes. Revue d'Economie Régionale et Urbaine, 2, p. 179-200.

Didier S., Peyroux E., Morange M., 2012. The spreading of the City Improvement District model in Johannesburg and Cape Town: Urban regeneration and the neoliberal agenda in South Africa. The International Journal of Urban and Regional Research, 36 (5), p. 915-935.

Gallet B., 2005. Les enjeux de la coopération décentralisée. Revue internationale et stratégique, 57, 1, p. 61-70.

Harris A., Moore S., 2013. Planning histories and practices of circulating urban knowledge. International Journal of Urban and Regional Research, 37, 5, p. 1499-1509.

Harrison P., 2015. South-south relationships and the transfer of 'best practice': the case of Johannesburg, South Africa. International Development Planning Review, p. 205-223.

Jacobs J. M., 2012. Urban geographies I: Still thinking cities relationally. Progress in Human Geography, 36(3): p. 412-422.

Knox P. L., Taylor P. J., (eds.) 1995. World cities in a world-system. Cambridge, Cambridge University Press, $348 \mathrm{p}$.

Knox P.L., Taylor P. J., 1995. World cities in a world-system. Cambridge University Press.

Kragelund P., 2011. Back to BASICs? The Rejuvenation of Non-traditional Donors' Development Cooperation with Africa. Development and Change, 42, p. 585-607.

Küber, D., Piliutyte J., 2007. Intergovernmental relations and international urban strategies: constraints and opportunities in multilevel polities. Environment and Planning C: Government and Policy, 25, 3, p. 357-373.

Lungkvist K., 2014. The Global City: From Strategic Site to Global Actor. In Curtis, S. (ed), The Power of Cities in International Relations. London and NewYork, Routledge, p. 32-55.

Massey D., 2005. For space. Sage, London, 222 p.

Mawdsley E., 2012a. The changing geographies of foreign aid and development cooperation: contributions from gift theory. Transactions of the Institute of British Geographers, 37, p. 256-272. 
Mawdsley E., 2012b. From Recipients to Donors: The Emerging Powers and the Changing Development Landscape. London, Zed.

McCann E., 2013. Policy boosterism, policy mobilities, \& the extrospective city. Urban Geography, 34(1), p. 5-29.

McCann E., Ward K., 2011. Mobile Urbanism: City policymaking in the global age. Minnesota, University of Minnesota Press, 256 p.

McCann E., Ward K., 2012. Assembling urbanism: Following policies and 'studying through' the sites and situations of policy making. Environment and Planning A, 44, p. 42-51.

McCann E., Ward K., 2010. Relationality/territoriality: toward a conceptualization of cities in the world. Geoforum, 41, p. 175-184.

Michel B., 2015. Reclaiming the European City. Business Improvement Districts in Germany. Urban Affairs Review, 51 (1), p. 74-98. (gemeinsam mit Christian Stein).

Nijman J.E., 2009. The rising influence of urban actors. Amsterdam Center for International Law (ACIL). The Broker, 17, p. 13-17.

Parnell S., Robinson J., 2012. (Re)theorizing cities from the Global South: looking beyond neoliberalism. Urban Geography, 33(4), p. 593-617.

Pasquier R., 2012. Quand le local rencontre le global : contours et enjeux de l'action internationale des collectivités territoriales. Revue française d'administration publique, 1, 141, p. 167-182.

Peck J., Theodore N., 2010. Mobilizing Policy: Models, Methods, and Mutations. Geoforum, 41, p. 169-174.

Peyroux E., 2015. Discourse of urban resilience and "inclusive development" in the Johannesburg Growth and Development Strategy 2040. The European Journal of Development and Research, Special issue on « Inclusive Development », 27 (4), p. 560-573.

Pinson G., Vion A., 2000. L'internationalisation des villes comme objet d'expertise. Pôle Sud, 13, p. 85-102.

Pirie G., 2010. Trajectories of North-South City Inter-relations: Johannesburg and Cape Town, 1994-2007. Urban Studies, 47, 9, p. 1985-2002.

Robinson J., 2011. Cities in a world of cities: the comparative gesture. International Journal of Urban and Regional Research, 35(1), p. 1-23.

Ruffin F.A., 2013. Municipal International Relations: The South African Case of Metropolitan eThekwini, Loyala. Journal of Social Sciences, XXVII, 1, Jan-June, p. 119-141.

Söderström O., 2014. Cities in Relation. Trajectories of urban development in Hanoi and Ouagadougou. Oxford, Wiley-Blackwell.

Söderström O., Dupuis B., Leu P., 2013. Translocal urbanism: how Ouagadougou strategically uses decentralised cooperation. In B. Obrist et al., Living the African City. Basel, SGAS (with Lit Verlag), p. 99-118.

van der Pluijm R., avec Melissen, J., 2007. City Diplomacy: The expanding role of cities in international politics. Netherlands Institute of International Relations, Clingendael Diplomatie Paper $n^{\circ} 10$.

van Ewijk E., Baud I., 2009. Partnerships between Dutch municipalities and municipalities in countries of migration to the Netherlands; knowledge exchange and mutuality. Habitat International, 33, 2, p. 218-226. 
Verdeil E., 2005. Expertises nomades au Sud. Éclairages sur la circulation des modèles urbains. Géocarrefour, 80 (3), p. 165-169.

Véron J.B., 2013. Les BRICS en Afrique : ambitions et réalités d'un groupe d'influence. Afrique contemporaine, $\mathrm{n}^{\circ} 248,4$, p. 7-9.

Viltard Y., 2008. Conceptualiser la 'diplomatie des villes' ou l'obligation faite aux relations internationales de penser l'action extérieure des gouvernements locaux. Revue française de science politique, 3, 58, p. 511-533.

Viltard Y., 2020. Diplomatie des villes : collectivités territoriales et relations internationales. Politique étrangère, 3, p. 593-604.

Ward K., 2006. "Policies in motion," urban management and state restructuring: The trans-local expansion of Business Improvement Districts. International Journal of Urban and Regional Research, Vol. 30, p. 54-75.

Wood A., 2014. Moving policy: global and local characters circulating bus rapid transit through South African cities. Urban Geography, 35(8), p. 1-17.

\section{NOTES}

1. Des entretiens ont été conduits auprès de quatre fonctionnaires de la Municipalité de Johannesburg impliqués dans la formulation et la mise en œuvre de la Stratégie des Relations Internationales (issus de l'ancien Department of Municipal International and Inter-governmental Relations, de la Central Strategy Unit, du Jo'burg Innovation \& Knowledge Exchange) et d'un consultant impliqué dans la mise en œuvre du partenariat entre Johannesburg et Addis-Abeba.

2. Il existe peu d'études sur les partenariats en cours à Johannesburg et la mise en œuvre de cette stratégie. Un recensement et une analyse des jumelages des villes et des provinces sud-africaines ont été conduits au milieu des années 2000 sans traiter d'études de cas (de Villiers, 2005). Des travaux ont été publiés sur les partenariats Nord/Sud entre l'Afrique du Sud les Pays-Bas (Bontenbal, 2009 ; Ewijk et Baud, 2009). Plus récemment le cas sud-africain a été traité à travers une étude de la politique des relations municipales de la ville d'eThekwini (Ruffin, 2013) et une analyse des partenariats Sud-Sud et des transferts de «bonnes pratiques» du point de vue de Johannesburg, resitués dans les dynamiques de transformation de la ville depuis les années 1990 (Harrison, 2015).

3. L'internationalisation des villes est un phénomène ancien, inscrit dans le processus-même de production de la ville et ce, dans toutes ses dimensions (économique, architecturale, urbanistique). La circulation internationale des politiques urbaines renvoie également à une histoire longue (Harris et Moore 2013). Nous nous intéressons ici à la littérature la plus récente conjuguant un intérêt pour les processus de circulation actuels et de nouvelles approches relationnelles des villes (Jacobs, 2012 ; Söderström, 2014).

4. Des travaux récents issus de la science politique ont réintroduit le rôle de l'Etat dans l'analyse des circulations de politiques publiques entre villes à l'échelle nationale et locale (Béal, Epstein et Pinson, 2015).

5. Les publications sur la «para-diplomatie » portent plutôt sur d'autres échelons administratifs (provinces dans le cas du Canada par exemple). Les travaux sur les "politiques municipales étrangères ", notamment aux Etats-Unis, incluent de leur côté les acteurs de la société civile.

6. Il s'agit des membres du Comité d'Aide au Développement de l'OCDE qui comprend 29 pays membres principalement des pays industrialisés du Nord. Ils sont tenus d'appliquer les recommandations adoptées par le CAD pour l'élaboration de leurs politiques nationales de 
coopération pour le développement (http://www.oecd.org/fr/cad/lesmembresducad.htm). Les pays dits émergents n'ont font pas partie.

7. Sous le régime de l'apartheid, l'État sud-africain a été exclu de plusieurs organismes internationaux (Organisation Mondiale de la Santé, Bureau International du Travail, Comité International Olympique), a fait l'objet de sanctions internationales (embargo sur les ventes d'armes et sur les produits pétroliers), de restrictions diplomatiques, de campagnes de boycott et de désinvestissement de la part des États occidentaux.

8. Pour une analyse plus détaillée du cadre institutionnel et légal qui régit les relations internationales en Afrique du Sud, voir Ruffin 2013.

9. Harrison (2015) indique de son côté que la municipalité de Johannesburg a adopté sa première politique des Relations Municipales Internationales en 2002 sans préciser les liens avec le cadre national de la MIR.

10. Pour plus de détails sur les échanges entre villes, voir Harrison, 2015.

11. Entretien avec un fonctionnaire de la CSU: Politiques et stratégies, Johannesburg, le 20 septembre 2011. Entretien 1.

12. Dans la suite du texte, les termes de "Municipalité de Johannesburg " et "Johannesburg» sont utilisés pour désigner l'acteur institutionnel qui a formulé et met en œuvre la stratégie des relations internationales. Comme l'analyse se fonde sur l'étude du document politique, le positionnement et les discours rapportés sont ceux des fonctionnaires de la CSU, sachant que le document a été approuvé par le cabinet du Maire.

13. Entretien 1.

14. Entretien 1.

15. Entretien avec un fonctionnaire de la CSU: Politiques et stratégies, Johannesburg, le 21 octobre 2013. Entretien 2.

16. Entretien avec un fonctionnaire de la CSU : Direction, Johannesburg, le 29 septembre 2011. Entretien 3.

17. Entretien avec un fonctionnaire du Jike : Knowledge Exchange Programme, Johannesburg, le 13 septembre 2011. Entretien 4.

18. Entretien avec un consultant indépendant, Johannesburg, le 21 septembre 2011. Entretien 5.

19. Entretien 1.

20. International Guangzhou Award for Urban Innovation: http://www.citiesalliance.org/joburglilongwe-guangzhou-award

21. Entretien avec un fonctionnaire de l'ex-External Relations Unit, Johannesburg, le 11 septembre 2011. Entretien 6.

22. L'Afrique du Sud ait acquis une expérience dans ce domaine avec la Coupe du Monde de Football 2010.

23. Entretien 6.

\section{INDEX}

Subjects: Sur le Champ - Sur le Terrain 
AUTHOR

ELISABETH PEYROUX

Élisabeth Peyroux, elisabeth.peyroux@cnrs.fr, est Chargée de recherche CNRS, membre de l'UMR 8586 Prodig. 